\title{
DESCONFORTOS VIVENCIADOS POR MULHERES SUBMETIDAS A HISTERECTOMIA
}

\author{
Ana Sofia Silva Veloso Amaral \\ Enfermeira no Centro Hospitalar de Trás-os-Montes e Alto Douro EPE \\ Mestre em Enfermagem de Saúde Materna e Obstétrica - Portugal anasofiadasilvaveloso@gmail.com \\ Emília Coutinho \\ UICISA:E / SIGMA - Phi Xi Chapter / CI\&DETS / Politécnico de Viseu - Portugal, ecoutinhoessv@gmail.com \\ Paula Nelas \\ UICISA:E / CI\&DETS / Politécnico de Viseu - Portugal \\ Cláudia Chaves \\ Doutoranda em Ciências de Enfermagem, Instituto de Ciências Biomédicas Abel Salazar / SIGMA - Phi Xi \\ Chapter / CI\&DETS / Politécnico de Viseu - Portugal \\ João Duarte \\ UICISA:E / CI\&DETS / Politécnico de Viseu - Portugal \\ Carla Cruz \\ UICISA:E / CI\&DETS / Politécnico de Viseu - Portugal
}

Recepción Artículo: 19 octubre 2020

Admisión Evaluación: 25 octubre 2020

Informe Evaluador 1: 25 noviembre 2020

Informe Evaluador 2: 23 noviembre 2020

Aprobación Publicación: 27 noviembre 2020

\section{RESUMO}

Enquadramento: 0 confronto com a necessidade de ter que se submeter a histerectomia nem sempre é fácil por parte da mulher/casal. Diferentes problemas vivenciados, poderão empurrar para essa decisão, mesmo pairando no andar dúvidas e preocupações quanto ao que poderá vir a sentir pós-histerectomia. Objetivos: conhecer os desconfortos vivenciados pelas mulheres que se submetem a histerectomia. Metodologia: Estudo exploratório e descritivo de natureza qualitativa. Foram participantes dez mulheres submetidas a histerectomia pertencentes a uma Unidade de Cuidados de Saúde Primários da Região Norte de Portugal. Recorreu-se à entrevista semiestruturada, no último trimestre de 2018, tendo-se procedido à transcrição do verbatim das entrevistas e análise qualitativa dos dados, como proposto por Bardin, com ajuda do QSR NVivo na sua versão 12, software de investigação qualitativa. Foi obtida a autorização para a realização do estudo por parte de Comissão de Ética e cumprido todos os procedimentos éticos exigidos a um estudo desta natureza. Os dados apresentados enquadram-se num estudo mais alargado no âmbito das vivências das mulheres submetidas a histerectomia. Resultados: Apresentam-se três categorias emergentes: "Problemas apresentados pelas mulheres antes da histerectomia" constituída por nove subcategorias sendo as hemorragias, os tumores, e o risco de cancro os mais referidos; "Desconfortos manifestados pelas mulheres após a histerectomia" constituída por seis subcategorias 


\section{DESCONFORTOS VIVENCIADOS POR MULHERES SUBMETIDAS A HISTERECTOMIA}

das quais a secura vaginal, dor na relação sexual e alteração da imagem corporal sobressaem; e a categoria "Atitudes dos enfermeiros valorizadas pelas mulheres", com quatro subcategorias, incluindo ajudarem a ultrapassar e tratarem bem Conclusões: se por um lado a histerectomia resolve problemas ginecológicos apresentados pela mulher, aumentado 0 seu bem-estar e qualidade de vida, por outro também a confronta com o aparecimento de novos desconfortos, para os quais nem sempre se sentia preparada.

Palavras-Chave: histerectomia; problemas; desconfortos; vivência; mulher

\section{ABSTRACT}

Discomfort experienced by women submitted to hysterectomy. Background: The confrontation, by the woman/couple, of the need to undergo through an hysterectomy is not always easy.. Different problems experiencedmay push towards this decision, even though doubts and concerns about what you may experience after hysterectomy are still present. Objective: to know the discomfort experienced by women who undergo hysterectomy. Methodology: Exploratory and descriptive qualitative study. Ten women who underwent hysterectomy belonging to a Primary Health Care Unit in the Northern Region of Portugal participated. A semi-structured interview in the last quarter of 2018, was used, followed by the verbatim transcription of the interviews and qualitative data analysis, as proposed by Bardin, with the help of QSR NVivo in version 12, qualitative research software. The Ethics Committee authorized the study to be carried out and all ethical procedures required for a study of this nature were complied with. The data presented are part of a larger study in the context of the experiences of women undergoing hysterectomy. Results: Three emerging categories are presented: "Problems presented by women before the hysterectomy" consisting of nine subcategories, with haemorrhages, tumours, and the risk of cancer being the most mentioned; "Discomfort manifested by women after hysterectomy" consisting of six subcategories, of which vaginal dryness, pain during intercourse and altered body image stand out; and the category "Nurses attitudes valued by the women", with four subcategories, including helping to overcome and treating them well. Conclusions: if on the one hand hysterectomy solves gynaecological problems presented by women, increasing their well-being and quality of life, on the other hand it also confronts them with the appearance of new discomforts, for which they did not always feel prepared.

Keywords: hysterectomy; problems; discomfort; experience; woman

\section{INTRODUÇÃO}

A decisão ou aceitação na realização de uma histerectomia nem sempre é pacifica por parte da mulher/casal. 0 útero é um órgão associado à feminilidade, sexualidade e a capacidade reprodutiva das mulheres. A perda desse órgão, simboliza a perda da função o que recai física, psicológica e culturalmente sobre a mulher e a sua vida conjugal.

É por vezes uma decisão que se vai adiando na esperança de que os problemas se resolvam ou desapareçam. E os receios iniciais por vezes concretizam-se, e outras vezes fica-se surpreendido com 0 resultado obtido. Dependendo das suas perspetivas em relação à histerectomia, a recuperação pós-cirúrgica e a sua adaptação diferem de mulher para mulher. Há sempre uma certa dúvida do que poderá vir a seguir e se não será eventualmente pior do que o que já se tem. Podem emergir sentimentos de tristeza e de medo, diminuição do interesse em atividades de lazer e sociais, perda ou ganho de peso significativo, insónia, fadiga e em pensamentos de morte. Todas as mulheres reagem de maneira diferente e as suas reações são uma combinação de respostas emocionais e físicas, para as quais os enfermeiros devem estar despertos. Vários estudos dão conta desta dualidade e diferentes respostas por parte das mulheres/casais.

Álvaro e Angelica (2017), através da aplicação da Escala Female Sexual Function Index, observaram que a maioria das mulheres apresentavam disfunção sexual. Estimou-se que a disfunção sexual é um fator de risco para um elevado agravamento da sua qualidade de vida, revelando as mulheres baixa autoestima. Verificaram também que a maioria das mulheres consideram a histerectomia como mutiladora, o que thes causa receios antes da cirurgia, contudo após a mesma encaram o procedimento como positivo para a saúde global. 
Teixeira e Batista (2016, p. 97) no seu estudo desocultaram as seguintes categorias: Satisfação pessoal após a cirurgia; Desmistificação da histerectomia; Indiferença do esposo diante da histerectomia; Mudança positiva na vida sexual; Importância da equipa no momento da cirurgia". Concluíram que, após a histerectomia, as mulheres tornaram-se mais resilientes e que a cirurgia Ihes proporcionou melhor qualidade de vida.

No pós cirurgia algumas mulheres confrontam-se com complicações que não contavam. Anjaneyulu, Kumar, Deepika, Alekhya e Harika (2016) fazem relatos de complicações imediatas pós-operatórias, como a febre, dor, hemorragia e retenção de urina, e ainda infeção da ferida. Quanto às complicações a longo prazo, referem as alterações do humor, dores musculares e articulares, incontinência, fraqueza e alteração do peso. Todavia, a maioria das mulheres percecionou a cirurgia como positiva, referindo que esta contribuiu para a melhoria da sua qualidade de vida, uma vez que deixaram de viver sem os transtornos causados pela patologia. Os mesmos autores referem que as vivências das mulheres após a histerectomia são únicas. Algumas ajustam-se melhor às mudanças que o seu corpo atravessa, enquanto outras podem experienciar uma série de emoções negativas, como a mutilação de uma parte do seu corpo, particularmente as mulheres com vida sexualmente ativa, resultando em estados depressivos.

Bossick, Sangha, Olden, Alexander e Wegienka (2018, p. 169) através de focus groups com mulheres com histerectomia deram conta da necessidade de se apostar num maior empowerment para que as mulheres possam vivenciar de forma mais positiva o período após cirurgia, juntamente com a transmissão de informações dadas para que estas tenham estejam preparadas para as mudanças somáticas que ocorrem pós-histerectomia. Neste sentido os profissionais de saúde ocupam um lugar privilegiado porquanto se acompanham a mulher/casal ao nível dos cuidados de saúde durante todo o processo. É fundamental apostar na formação destes profissionais de modo a que isso se reflita na qualidade dos cuidados prestados.

Monterrosa-Castro, Monterrosa-Blanco e Beltrán-Barrios (2018, pp. 102-105) verificaram que a insónia e a disfunção sexual são fatores preditores de redução da qualidade de vida em mulheres sexualmente ativas previamente e após a histerectomia.

Freitas, Gomes, Campos, Estrela, Cordeiro e Santos (2016, pp. 7-9), cuja revisão integrativa da literatura revela que os estudos analisados documentam que as vivências e sentimentos de algumas mulheres submetidas a histerectomia são de revolta, medo, tristeza, vazio, enquanto outras encaram a histerectomia como um alívio e com a esperança de resolução do seu problema.

Silva e Vargens (2016, pp. 3-7) constaram que após cirurgia as mulheres demonstram sentir-se diferentes, tendo a mutilação sido sentida como "concreta", devido à perda do órgão, e "abstrata", estando associada "ao impacto na identidade social e funcional feminina", sendo o corpo cultural e historicamente construído. Nesse sentido consideram ser fundamental o papel do enfermeiro em instituir "um cuidado multidimensional, que identifique as necessidades que vão além do corpo biológico".

Também Barbosa, Santos \& Rodrigues (2018, p. 227) através de uma revisão integrativa da literatura, analisaram a experiência de mulheres submetidas a histerectomia e verificaram que os desconfortos físicos resultam em fragilidade emocional por Ihes despertar sentimentos de doença e indisposição, levaram à aceitação da terapêutica, mesmo sendo um procedimento cirúrgico, em decorrência do desejo de se verem livres dos problemas que estão a vivenciar, percecionando a histerectomia como a "cura". Contudo, verificaram também que as mulheres, não obstante terem sido informadas acerca do diagnóstico que resultou em histerectomia, manifestaram um desconhecimento em relação ao significado da mesma. Esta constatação deixa-nos campo para reflexão de como, profissionais de saúde ainda temos um caminho a percorrer quer na compreensão do fenómeno quer nas estratégias de resposta a desenvolver com as mulheres/casais que são submetidos a histerectomia. Ficou-nos a questão: Quais os desconfortos vivenciados pelas mulheres submetidas a histerectomia?

\section{METODOLOGIA}

Estudo exploratório e descritivo de natureza qualitativa. Neste estudo partiu-se da seguinte questão de investigação: quais os desconfortos vivenciados pelas mulheres submetidas a histerectomia? Nesse sentido, foram 


\section{DESCONFORTOS VIVENCIADOS POR MULHERES SUBMETIDAS A HISTERECTOMIA}

participantes no estudo dez mulheres submetidas a histerectomia pertencentes a uma Unidade de Cuidados de Saúde Primários da Região Norte de Portugal. Recorreu-se à entrevista semiestruturada, no último trimestre de 2018. As entrevistas decorreram em local especifico para o efeito sendo um espaço reservado que permitiu que as mulheres falassem livremente sobre a problemática. As entrevistas foram gravadas, após obtenção do consentimento por parte das mulheres. Os testemunhos das mulheres foram transcritos, tendo-se procedido de seguida à análise qualitativa dos dados, como proposto por Bardin (2015), com ajuda do QSR NVivo na sua versão 12, software de investigação qualitativa. A análise de conteúdo é composta por um conjunto de técnicas de análise dos discursos das participantes, que se baseiam em procedimentos sistemáticos e objetivos de descrição do conteúdo das mensagens, tendo sido nossa opção a análise temática como proposto por Bardin (2015). Por sua vez, o Nvivo® é uma das aplicações usadas na análise de discurso, teoria fundamentada, análise de conversação, etnografia, revisões de literatura, fenomenologia e métodos mistos entre outros e que para além de outras possibilidades, permite organizar, classificar, ordenar, examinar relações e combinar análises.

Todos os procedimentos éticos inerentes à investigação foram cumpridos, tendo-se obtido a autorização para a realização do estudo por parte de Comissão de Ética, bem como o consentimento informado por parte das participantes depois de Ihes ter sido apresentado o estudo e obtida a manifestação da sua aceitação nesta participação.

Durante a após a análise dos dados foi mantida a sua anonimização, não sendo possível em circunstância alguma identificar as participantes. Adotamos a codificação das entrevistadas assumindo a letra $E$ de entrevistada seguida de número árabe de 1 a 10 em função da entrevistada a que se refiram os dados.

Os dados aqui apresentados enquadram-se num estudo mais alargado no âmbito das vivências das mulheres submetidas a histerectomia.

\section{RESULTADOS}

A categoria "Problemas apresentados pelas mulheres antes da histerectomia" é constituída por nove subcategorias. As subcategorias mais referenciadas pelas mulheres foram: "Hemorragias" com oito unidades de registo, "Tumores" com oito unidades de registo, "Risco de cancro" com oito unidades de registo. Menos referidas pelas mulheres foram as subcategorias: "Anemia" com quatro unidades de registo, "Dor" com duas unidades de registo, "Cancro" com quatro unidades de registo, "Prolapso uterino" com duas unidades de registo, "Sofrimento" com duas unidades de registo e Quistos nos ovários com uma unidade de registo (cf. tabela 1).

Tabela 1 - Problemas apresentados pelas mulheres antes da histerectomia

\begin{tabular}{lcc}
\hline $\begin{array}{l}\text { Problemas apresentados pelas mulheres } \\
\text { antes da histerectomia }\end{array}$ & n & Ur \\
\hline Hemorragias & 6 & 8 \\
Tumores & 5 & 8 \\
Risco de cancro & 3 & 8 \\
Anemia & 2 & 4 \\
Dor & 2 & 2 \\
Cancro & 1 & 4 \\
Prolapso uterino & 1 & 2 \\
Sofrimento & 1 & 2 \\
Quistos nos ovários & 1 & 1 \\
\hline
\end{tabular}

A categoria "Desconfortos manifestados pelas mulheres após a histerectomia" é constituída por seis subcategorias. As subcategorias mais referenciadas pelas mulheres foram: "Secura vaginal" com seis unidades de registo; "Alteração da imagem corporal" com três unidades de registo; "Dor na relação sexual" com três unidades 
de registo; "Engordar" com três unidades de registo" e "Entrar na menopausa" com duas unidades de registo. A subcategoria "Afrontamentos" foi a menos referenciada com uma unidade de registo (cf. tabela 2).

Secura vaginal

"às vezes sinto a minha vagina um bocadinho seca" (E1)

"Sinto-me que não tenho aquela humidade na minha vagina como antes" (E1)

"Tenho secura vaginal" (E5)

"Também fiquei com secura vaginal" (E7).

Alteração da imagem corporal

"porque eu tive de me mentalizar, eu sozinha com o meu corpo, conforme ficou" (E1)

"A cicatriz foi feita no mesmo sítio onde fizeram a cesariana - só não gostei muito no início de ver a cicatriz. Mas isso é secundário. É uma questão de estética que quase já normalizou" (E4)

Dor na relação sexual

"Cada vez que há relações sexuais sofro bastante porque tenho muitas dores" (E6), "Não sei se é por sentir dores" (E8)

"por causa da dor" (E8)

Engordar

"comei a engordar mais um bocadinho" (E1)

"Eu sofri um bocado porque engorder" (E6)

"E mesmo com a alimentação que eu fazia e tudo, mas engordei bastante, 8/10 kg, lentamente mas engorde" (E6)

Entrar na menopausa

"sofri porque também entrei na menopausa" (E6)

"entrei numa pré-menopausa" (E7)

Afrontamentos

"Depois fiquei com os afrontamentos e a médica deu-me uns selos, mas depois passou" (E10)

"a nível da roupa, eu não podia vestir umas calças normais, porque fazia-me mal-estar" (E9)

Tabela 2 - Desconfortos manifestados pelas mulheres após a histerectomia"

\begin{tabular}{lcc}
\hline $\begin{array}{l}\text { Desconfortos manifestados pelas } \\
\text { mulheres após a histerectomia }\end{array}$ & n & Ur \\
\hline Secura vaginal & 4 & 6 \\
Alteração da imagem corporal & 3 & 3 \\
Dor na relação sexual & 2 & 3 \\
Engordar & 2 & 3 \\
Entrar na menopausa & 2 & 2 \\
Afrontamentos & 1 & 1 \\
\hline
\end{tabular}

A categoria "Atitudes dos enfermeiros valorizadas pelas mulheres" é constituída por quatro subcategorias, todas referenciadas apenas por uma mulher cada. A subcategoria "Ajudaram-me a ultrapassar" tem duas unidades de registo, as restantes, "Foram espetaculares", "Foram impecáveis" e "Trataram-me bem", têm todas apenas uma unidade de registo (cf. tabela 3).

Ajudaram-me a ultrapassar

"Cada vez que tinha que lá ir ela (enfermeira) conversava muito comigo. Dizia que agora era outra nova vida e que ficava aliviada e que era o melhor para mim. E de facto, o que ela me dizia assim foi", "Eles (enfermeiros) ajudaram muito, muito no ultrapassar esta fase (E7) 


\section{DESCONFORTOS VIVENCIADOS POR MULHERES SUBMETIDAS A HISTERECTOMIA}

Foram espetaculares

"os enfermeiros foram espetaculares" (E7)

Foram impecáveis

"eles [enfermeiros] foram impecáveis" (E8)

Trataram-me bem

"trataram-me lá muito bem. Fui muito bem tratada tanto a nível de enfermeiras ou auxiliares. Fui lá muito bem tratada" (E9)

Tabela 3 -Atitudes dos enfermeiros valorizadas pelas mulheres

\begin{tabular}{lcc}
\hline $\begin{array}{l}\text { Atitudes dos enfermeiros valorizadas pelas } \\
\text { mulheres }\end{array}$ & $\mathbf{n}$ & $\mathbf{U r}$ \\
\hline Aiudaram-me a ultrapassar & 1 & 2 \\
Foram espetaculares & 1 & 1 \\
Foram impecáveis & 1 & 1 \\
Trataram-me bem & 1 & 1 \\
\hline
\end{tabular}

\section{DISCUSSÃO DOS RESULTADOS}

A categoria problemas apresentados pelas mulheres antes da histerectomia foi referenciada por todas as participantes. Os problemas que mais referenciaram foram: "Hemorragias", com oito unidades de registo. Os problemas apontados pelas mulheres antes da histerectomia estão registados na literatura, segundo a qual, as principais indicações para a sua realização partem do prolapso de órgãos pélvicos, leiomiomas uterinos, dor ou infeção pélvica, prolapso uterino, sangramento uterino anormal e doenças malignas e pré-malignas (Cardoso et al., 2017, p.18).

No que se refere aos "Desconfortos manifestados pelas mulheres após a histerectomia" verificou-se que os mesmos foram referenciados por oito mulheres, correspondendo a 18 unidades de registo, tendo a subcategoria "Afrontamentos" sido referenciada por uma mulher. A subcategoria "Alteração da imagem corporal" foi apresentada por três mulheres, com uma unidade de registo cada. De acordo com duas mulheres, após a histerectomia, ficaram com dor na relação sexual, o que se constituiu como subcategoria com três unidades de registo. De acordo com 2 mulheres, um dos desconfortos da histerectomia foi o facto de engordarem, uma subcategoria com 3 unidades de registo. A subcategoria "Entrar na menopausa" foi referenciada por 2 mulheres. Por último, 4 mulheres referiam, como desconforto, a "Secura vaginal", uma subcategoria com 6 unidades de registo. Os desconfortos mencionados pelas mulheres são concordantes com a literatura consultada, sendo exemplo o estudo de Álvaro e Angelica (2017), no qual a maioria das mulheres apresentava disfunção sexual, secura vaginal, alteração da sua imagem corporal, sobretudo porque aumentaram de peso, e algumas delas entram numa menopausa precoce. Barbosa, Santos e Rodrigues (2018, pp. 227-228) referem que estas mulheres experienciam desconfortos psicossociais e físicos que resultam em fragilidade emocional. As mesmas autoras acrescentam que, deste modo, é necessária uma atenção especial em relação à histerectomia porque a mesma, geralmente, resulta em sofrimento, dúvidas e receios, baseados "em crenças e valores que podem levar a reformulações ligadas ao feminino, à representação social, ao género, à autoimagem, à sexualidade e à relação conjugal e social". Sendo uma cirurgia irreversível, resulta, em muitos casos, em alterações da integridade corporal da mulher, causando-Ihes desconfortos, não devendo, por isso, ser banalizada pelos profissionais de saúde.

A categoria "Atitudes dos enfermeiros valorizadas pelas mulheres" foi uma das categorias com menos referências pelas mulheres. Das atitudes desocultadas pelos discursos das participantes emergem os enfermeiros ajudaram a ultrapassar a situação, foram espetaculares, foram impecáveis e trataram-me bem. Importa referir 0 facto de apenas quatro mulheres terem feito referência aos cuidados individualizados por parte dos enfermeiros, o que implica um reforço junto dos enfermeiros que prestam cuidados a estas mulheres, uma vez que, como refe- 
re Barros (2014), a histerectomia, por norma, desencadeia grandes mudanças na vida da mulher, devendo a abordagem por parte dos enfermeiros ser individualizada. É importante que os enfermeiros ajudem as mulheres a ajustarem-se melhor às modificações que 0 seu corpo atravessa, podendo, inclusive, experienciar um conjunto de emoções negativas, como a mutilação de uma parte do seu corpo, especialmente as mulheres com vida sexualmente ativa, o que pode resultar em estados depressivos (Anjaneyulu et al., 2016). Assim sendo, a assistência do enfermeiro nos cuidados pré e pós-operatório na mulher submetida a histerectomia deve ser individualizada e numa perspetiva holística, porque cada uma vivência esta experiência de acordo com a sua singularidade. Como enfatizam Gomes e Romanek (2013, p. 18) deve cuidar-se da mulher submetida a histerectomia "como um ser humano com sentimentos e necessidades humanas próprias".

\section{CONCLUSÃO}

Cada mulher é singular e, como tal, as suas vivências face à histerectomia também são singulares. 0 útero compreende uma parte importante da autoimagem da mulher e a sua perda significa a privação do senso de feminilidade, o que pode despoletar uma miríade de sentimentos. Neste sentido, os enfermeiros têm uma importante participação no cuidado e na orientação destas mulheres, para além de as informarem e tranquilizarem, tendo como principal objetivo a minimização do seu sofrimento psicológico. Para tal, é fundamental uma assistência diferenciada e individualizada, reconhecendo as suas necessidades de uma forma holística, para que possam ajudar as mulheres a adaptarem-se à sua nova condição de mulher, o mais positivamente possível.

Os resultados obtidos desocultam vários problemas apresentados pelas mulheres antes da histerectomia, nomeadamente: anemia, cancro, dor, hemorragias, prolapso uterino, quistos nos ovários, risco de cancro, sofrimento e tumores; realçam também vários desconfortos vivenciados pelas mulheres após a histerectomia, nomeadamente: afrontamentos, alteração da imagem corporal, dor na relação sexual, engordar, entrar na menopausa e secura vaginal; e desocultam algumas atittudes dos enfermeiros que foram valorizadas pelas mulheres na interação que com elas estabeleceram, considerando que eles souberam ajudar a ultrapassar, terem sido espetaculares, impecáveis e trataram bem.

Desocultar os significados que cada mulher atribuiu à sua experiência de vida, foi o desafio neste caminhar para a consciencialização na importância da formação dos enfermeiros para a capacitação da mulher que vivencia a histerectomia.

\section{REFERÊNCIAS BIBLIOGRÁFICAS}

Álvaro, M.-C., \& Angelica, M.-B. (2017). Sexual Dysfunction and Quality of Life in Colombian Histerectomized Women. Perceptions in Reproductive Medicine, 1(3), 2-3. PRM.000513. 2017. DOI: 10.31031/PRM.2017.01.000513

Anjaneyulu, G., Kumar, M., Deepika, P., Alekhya, \& Harika (2016). The profile of hysterectomized women from an urban slum of Hyderabad, Telangana state, India. International Journal of Community Medicine and Public Health Anjaneyulu G et al. Int J Community Med Public Health; 3(9), 2633-2636. Acedido em http://www.jicmph.com

Barbosa, A.R.S., Santos, A.N., \& Rodrigues, T.S. (2018). Experiência de mulheres que realizaram histerectomia: revisão integrativa. Rev. UNINGÁ, Maringá, v. 55, 2, 227-241. ISSN 2318-0579

Bardin, L. (2015). Análise de conteúdo. Lisboa: Edições 70.

Barros, A. S. (2014). Cuidados de enfermagem a pacientes Histerectomizadas: Revisão integrativa da literatura. (Bacharel ). Universidade de Brasília Brasília.

Bossick, A.S., Sangha, R., Olden, H., Alexander, G.L., \& Wegienka, G. (2018). Identifying What Matters to Hysterectomy Patients: Postsurgery Perceptions, Beliefs, and Experiences. Journal of Patient-Centered Research and Reviews, vol. 5, 2, 167-175. Acedido em https://digitalrepository.aurorahealthcare.org/cgi/viewcontent.cgi?article=1581\& context=jpcrr 
Cardoso, B.C., Cardoso, C.R., \& Fernandes, I.F. (2017). Perfil de Mulheres Submetidas a Histerectomia e Influência da Deambulação na Alta Hospitalar. Pleiade, 11(21), 17-24, Jan./Jun., 2017.

Freitas, B.F., Gomes, N.P., Campos, L.M., Estrela, F.M., Cordeiro, K.C.C., \& Santos, R.M. (2016). Complicações pós-cirúrgicas da histerectomia: revisão integrativa. Revista Baiana de Enfermagem, Salvador; v. 30, 2, 1-11.

Gomes, I.M., \& Romanek, F.A.R.M. (2013). Enfermagem perioperatória: cuidados à mulher submetida à histerectomia. São Paulo: Revista Recien.; 3(8), 18-24. Acedido em https://www.recien.com.br/index.php/Recien/article/view/53/151

Monterrosa-Castro, A., Monterrosa-Blanco, A., \& Beltrán-Barrios, T. (2018). Insomnia and sexual dysfunction associated with severe worsening of the quality of life in sexually active hysterectomized women. Sleep Sci.; 11(2), 99-105. DOl: 10.5935/1984-0063.20180019

Silva, C.M.C., \& Vargens, O.M.C. (2016). A mulher que vivencia as cirurgias ginecológicas: enfrentando as mudanças impostas pelas cirurgias. Rev. Latino- Am. Enfermagem; 24, 2-8.

Teixeira, M.R.,\& Batista, E.C. (2016). Vivências Cotidianas da Mulher Histerectomizada: Narrativas e Contextos. Rev Enfermagem e Saúde Coletiva. v. 1, 2, p. 91, Faculdade São Paulo - FSP. 